\title{
Design of Traceability and Forgery Prevention Management System for Agricultural Products Breeding
}

\author{
Zuliang Wang ${ }^{a^{*}}$ and Haoxiang Zhang ${ }^{\text {b }}$ \\ Department of Information Engineering, Xijing University, Xi'an, 710123, China \\ a1601252678@qq.com, b337907371@qq.com
}

\begin{abstract}
Keywords: Agricultural product breeding; Traceability; Anti-counterfeiting; Radio frequency identification
\end{abstract}

\begin{abstract}
In recent years, endless accidents of quality and safety of agricultural products have caused confusion and uneasiness among consumers. The problem of false seeds, which are harmful to farmers and governments at all levels, has always been the source of trouble to farmers. False seeds often lead to farmers' grain harvesting, and do great harm to society. In order to improve the quality of agricultural products, the electronic labels were made into high-imitation electronic seeds by means of radio frequency identification, Internet of Things and other information technologies for special commodities such as corn, wheat and rice. A high-imitation electronic seed was packed in each seed bag, and the electronic labels were coded to realize the anti-counterfeiting of seeds. Fake, anti fleeing, quality tracing, information collection, etc. The design of seed quality traceability and anti-counterfeiting system with the information chain of seed production and processing activities as the core can improve the standardization of seed production, establish a standardized market environment, and safeguard the vital interests of seed enterprises and agricultural growers.
\end{abstract}

\section{Introduction}

China is a big agricultural country, and the planting output of agricultural products accounts for a large proportion in China. Except for the planting technology and planting area, the most important factor is how to produce good quality and quantity of agricultural products. "One grain of millet is planted in spring and ten thousand seeds are harvested in autumn." The development of high-yield, high-quality and safe production of agriculture and animal husbandry is inseparable from fine plant seeds. Seeds are the basic means of production in agricultural production. Agriculture begins with sowing. Whether seeds can germinate, emerge and become seedlings after sowing is the basis of high yield and quality. Agricultural production is based on planting, and seed is the continuation and development of planting, is a special means of production with vitality. Seed quality, which affects the yield, quality and resistance of crops, is the internal factor to achieve high yield and quality of crops, and is the core carrier and key of technical measures.

"Agriculture is the foundation of the country and the foundation for planting". With the continuous development of the seed market, the problem of counterfeit and inferior seeds and the act of fleeing seeds have been happening, which has affected the normal development of agriculture. On the one hand, the formal enterprises have caused a large degree of losses and disrupted the normal circulation order of the seed market; on the other hand, they have deceived consumers, resulting in yield reduction and damage to consumers. Legitimate interests have posed a serious threat to the development of agriculture in China. Therefore, the design of traceability and anti-counterfeiting management system for agricultural products can improve the quality of seeds. Therefore, the design of traceability and anti-counterfeiting management system for agricultural products can improve the quality of seeds ${ }^{[1 \sim 3]}$.

\section{Traceability and Anti-counterfeiting Management System for Agricultural Products Breeding}

Current applications usually stick RFID tags on the packaging bags, and numerous profiteers can still drop the bags and string goods, which cannot fundamentally prevent the occurrence of false 
seeds. For special commodities such as corn, wheat and rice seeds, electronic labels can be made into highly imitative electronic seeds, and a high imitative electronic seed can be packed in each seed bag, and the electronic labels are coded to achieve anti-counterfeiting, anti-channeling, quality traceability, information collection and so on, thus effectively improving the production enterprises. Production efficiency and management level in warehousing and logistics links, enhance user trust and market confidence, enhance enterprise competitiveness and image, and protect enterprise reputation. The terminal anti-counterfeiting inquiry and covert anti-channeling inspection can be realized by using the non-contact reading characteristic of radio frequency identification (RFID), and the problems of easy imitation and destruction existing in the anti-counterfeiting and anti-channeling means of seed industry can be solved.

The Composition of the System. The system includes seed packaging bags, real seeds, highly bionic electronic seeds embedded with radio frequency tags, and readers. Seed packaging bags for plastic bags, paper bags can be used to pack seeds; real seeds for all anti-counterfeiting traceability of high-quality seeds; embedded in radio frequency electronic tags of high-imitation electronic seeds and real seeds mixed, with the naked eye cannot be identified, to prevent dropping, stringing; reader for writing or reading electronic tag information.

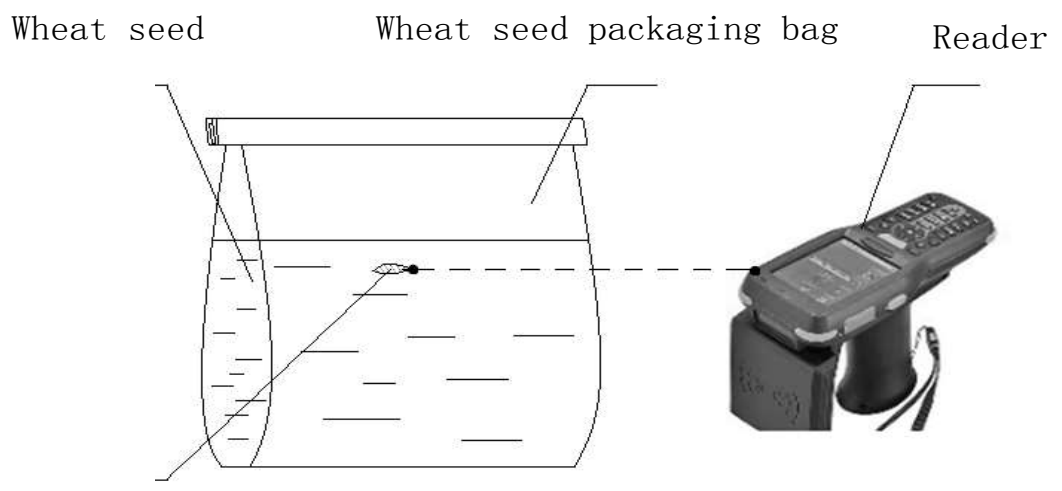

High imitation electronic wheat grain embedded with RFID tag

Figure1. The Schematic diagram

Among them, the seed packaging bag is the smallest anti-counterfeiting traceability management unit, each bag is filled with pre-determined weight seeds, and each bag is filled with a high-imitation electronic seeds embedded with radio frequency tags. True seeds are good seeds for anti-counterfeiting. The highly imitated electronic seed embedded in the RFID tag is an electronic tag encapsulated in the shape and color of a seed, which is guaranteed to be unrecognizable to the naked eye to prevent dropping and stringing. The IP68 package can be waterproof, dustproof, anti falling and anti smashing to ensure firmness and environmental adaptability. The reader is used to write or read the tag information. UHF reader is used. The communication distance between the reader and the high-imitated electronic wheat can reach more than 10 meters. ISO18000-6 standard is adopted to support the batch reading and writing operation of the reader for multi-tag, and to support the reading and writing in motion to provide automatic storage, logistics, distribution and other management. To improve breeding management efficiency.

The Working Principle of the System. Agricultural products breeding traceability anti-counterfeiting management system, seeds into packaging bags, embedded in electronic tags of high-imitation seeds and seeds mixed; Reader and embedded in electronic tags of high-imitation seeds wireless connection for writing or reading electronic tag information; packaging bags made of unshielded electrical signal materials; embedded electronic tags High imitation seeds and seeds can not be distinguished from the naked eye, and are used to prevent dropping and exchanging goods. The working principle of the system is shown in Figure 2. 


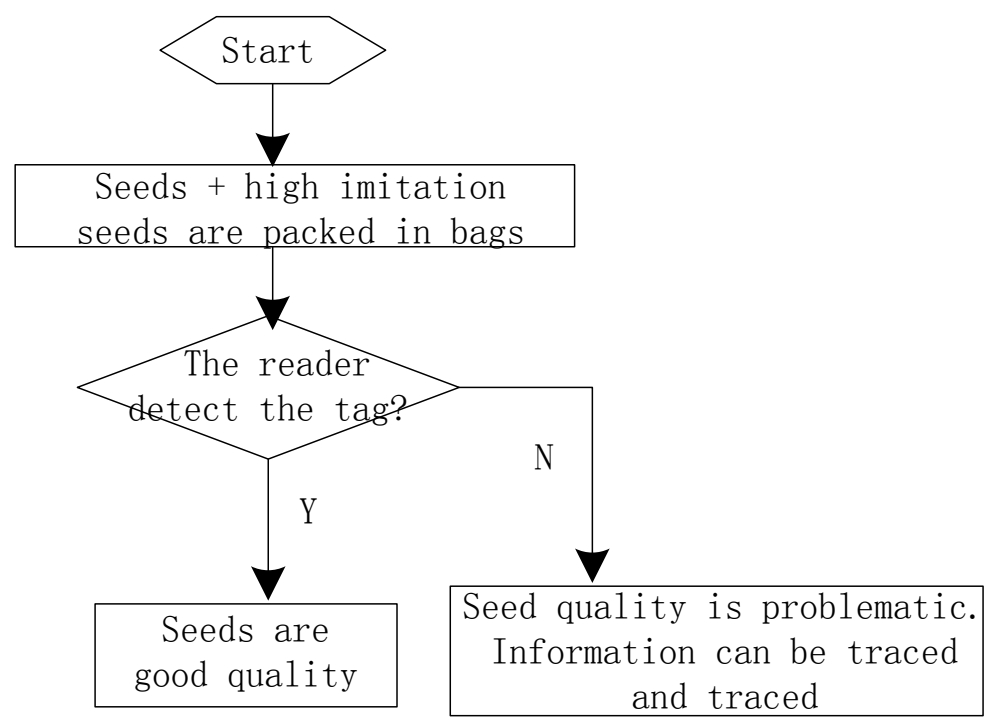

Figure 2. The working principle of the system

When the reader detects the tags in the highly imitated seeds, it indicates the authenticity of the seeds. Otherwise, the seeds may appear to be false. Effectively improve the production efficiency and management level of enterprises in warehousing and logistics links, enhance user trust and market confidence, enhance the competitiveness and image of enterprises, and protect the reputation of enterprises. The terminal anti-counterfeiting inquiry and covert anti-channeling inspection can be realized by using the non-contact reading characteristic of radio frequency identification (RFID), and the problems of easy imitation and destruction existing in the anti-counterfeiting and anti-channeling means of seed industry can be solved.

\section{Summary}

The traceability and anti-counterfeiting management system of agricultural product breeding makes use of radio frequency identification technology to make electronic tags into high-imitation electronic seeds. In each seed packaging bag, a high-imitation electronic seed is loaded and the electronic tags are coded. Once the seeds have been replaced by bad businessmen, we can detect the false seeds placed in the seeds in advance to achieve anti-counterfeiting, anti-fleeing goods, quality traceability, information collection and so on. Ensure the quality of seeds, avoid fake seeds and improve the quality of agricultural products.

\section{Acknowledgements}

This study was funded by Key Research and Development Plan Project of Shaanxi Provincial Science \& Technology Department (Program No. 2018ZDXM-NY-014).

\section{References}

[1] Chen Ying, Zhang Youhua, Guo Shupu, Lu Xuzhong. Design and implementation of wheat seed anti-counterfeiting early warning system based on intelligent packaging [J].Journal of Anhui Agricultural University, 2016, 43 (06): 1033-1038.

[2] Chen Ying. Study on traceability, anti-counterfeiting and early warning of wheat seeds based on seed identity cards [D]. Anhui Agricultural University, 2016.

[3] Zhang Liping. Research and Application of Seed Traceability and Supervision System Based on Crop Variety Identity Card [D]. Anhui Agricultural University, 2016.

[4] Bao Yuting, Chen Xuefeng, Zhang Li. Differential Diagnosis of Dairy Cow Ruminant Disorder [J].Agricultural Development and Equipment, 2016, (01): $164+159$. 
[5] Schirmann K, Wujiangli. Changes in rumination and feeding behavior of dairy cows before and after calving [J].Chinese Animal Husbandry Veterinary, 2013, 40 (12): 84.

[6] Feng Tao, Guo Yong, Bai Jiahua, Xu Xiaoling, Liu Yan.A new estrus identification technique based on cow rumination time[J].Chinese Animal Husbandry Veterinary, 2013, 40 (07): 198-200.

[7] Gang Li. Prevention and Control of Peste des Petits Ruminants in China[A]. International Society of Zoological Sciences.Abstracts of the 7th International Symposium of Integrative Zoology__Wildlife Monitoring and Data Collection under Global Change[C].International Society of Zoological Sciences,2015:1.

[8] Song Huaibo, Li Tong, Jiang Bo, Wu Qian, He Dongjian.Multi-objective automatic monitoring of ruminant cow's mouth based on Horn-Schunck optical flow[J].Journal of Agricultural Engineering, 2018,34(10): 163-171.

[9] Qiu Wenqing, Wang Yachun, Huang Xixia. Knowledge Mapping Analysis of World Livestock Ruminant Behavior Research [J].Heilongjiang Animal Husbandry Veterinary, 2018 (05): 97-103+263-264.

[10] Zhang Shuang. Research on individual ruminant behavior monitoring technology in [D]. [D]. Northeast Agricultural University, 2017.

[11] Fu Yao, Wang Yajing, Cao Zhijun, Li Shengli. Research Progress on Factors Affecting DairyCows'Feeding Behavior [J].Chinese Journal of Animal Husbandry, 2015, 51 (20): 71-75.

[12] Feng Jun, Xie Zhenming, Ding Yi, Wujiang, Yang Chunying, Zhao Xinhua, Li Xia, Chen Hongdi. The necessity of seed traceability and the realization of two-dimensional code technology [J].Agricultural Science and Technology Communication, 2015 (10): 7-8+12. 